\title{
Scalp Leiomyosarcoma: Diagnosis and Treatment During a Global Pandemic With COVID-19
}

\author{
Hebah Hassan a, b, Amit Elazar ${ }^{\mathrm{a}}$, Kazuaki Takabec, d, e, f, g, Rajiv Datta ${ }^{\mathrm{a}}$, \\ Hideo Takahashi ${ }^{\mathrm{a}}$, Eric Seitelman ${ }^{\mathrm{a}, \mathrm{h}}$
}

\begin{abstract}
Leiomyosarcoma is an aggressive, uncommon sarcoma effecting smooth muscle tissue. Prompt tissue diagnosis and staging workup are keys to preventing distant metastasis. Identification of this rare sarcoma has become increasingly difficult with decreased ability to seek out non-coronavirus disease 2019 (COVID-19) medical care. The pandemic has caused a widespread healthcare demand with providers reaching their full capacity causing care and resources to be shifted to the pandemic. We have experienced an 83-year-old male who significantly delayed to seek any medical attention for his scalp lesion for several months due to a combination of fear and decreased available appointments. Since the patient presented with a delayed scalp leiomyosarcoma, he required an extensive excision and flap reconstruction for the lesion. This case sheds light on the importance of weighing the risks and benefits associated with cancer management during the pandemic for both patients and healthcare providers. The healthcare system's response to the pandemic also played a role in this case as well, with shorter appointment times and decreased frequency of follow-up. As a result, the pandemic has had a catastrophic impact on the diagnostic pathway for cancer. This case report discusses the difficulties in diagnosing and treating a rare cancer such as scalp leiomyosarcoma amidst the global pandemic and the importance of telemedicine in improving future outcomes.
\end{abstract}

Keywords: Scalp leiomyosarcoma; COVID-19; Pandemic; Telemedicine

Manuscript submitted May 21, 2021, accepted June 2, 2021

Published online July 10, 2021

aDepartment of Surgery, Mount Sinai South Nassau, Oceanside, NY, USA

${ }^{b}$ New York Institute of Technology College of Osteopathic Medicine, Old Westbury, NY, USA

'Department of Surgical Oncology, Roswell Park Comprehensive Cancer Center, Buffalo, NY, USA

${ }^{\mathrm{d}}$ Department of Surgery, University at Buffalo Jacobs School of Medicine and Biomedical Sciences, The State University of New York, Buffalo, NY, USA

eDepartment of Breast Surgery and Oncology, Tokyo Medical University, Tokyo, Japan

fDepartment of Surgery, Yokohama City University, Yokohama, Japan

gDepartment of Surgery, Niigata University Graduate School of Medical and Dental Sciences, Niigata, Japan

${ }^{\mathrm{h}}$ Corresponding Author: Eric Seitelman, Department of Surgery, Mount Sinai South Nassau, Oceanside, NY, USA. Email: Eric.Seitelman@snch.org

doi: https://doi.org/10.14740/wjon1393

\section{Introduction}

Leiomyosarcoma is a rare soft tissue sarcoma, consisting of $1 \%$ of malignant tumors, typically found in the uterus, gastrointestinal tract, and retroperitoneum [1]. It is even rarer to encounter a superficial leiomyosarcoma in clinical practice, as they consist of $7-10 \%$ of diagnosed sarcoma cases. Leiomyosarcoma is known to be locally aggressive and difficult to diagnose, emphasizing the necessity for prompt diagnosis and treatment [2]. Although diagnosis and treatment for rare cancers have already been difficult, it has become increasingly challenging due to the coronavirus disease of 2019 (COVID-19) pandemic.

Amidst the COVID-19 pandemic, there have been profound modifications in the healthcare field to minimize virus spread. The pandemic has overwhelmed the healthcare system capacity in the USA, leading to significant care postponement [3]. This, in combination with patient reluctance towards seeking medical attention due to fear of contracting COVID-19, may have a detrimental effect on timely diagnosis and treatment. As with other malignancies, early diagnosis of sarcoma is crucial as prognosis is dependent on stage at diagnosis [4]. The purpose of this report is to discuss both diagnosis and management of leiomyosarcoma in the scalp to aid physicians navigating cancer care during the pandemic.

\section{Case Report}

\section{Investigations}

An 83-year-old male with no significant past medical history presented to the clinic with a large, fungating scalp mass. The patient struck his head on an open cabinet door but did not seek immediate medical care as he did not have additional symptoms. He also avoided going to a physician's office as the pandemic was on the rise. The patient presented to his primary care physician (PCP) for the first time approximately 4 weeks after the initial injury, for a persistent "lump on his head." The mass was approximately $2 \mathrm{~cm}$, fluctuant and friable with recurrent episodes of bleeding on the left aspect of frontal head above the left eyebrow.

\section{Diagnosis}

This was initially diagnosed as a hematoma of the scalp, and he 
was advised to avoid self-manipulation, and to hold his aspirin at this time. Subsequently he was referred to a wound care clinic for possible drainage, where local wound care with a soft absorbent dressing consisting of a hydrophilic polyurethane foam sheet bonded to a semipermeable polyurethane film, with dry dressing was recommended. Due to the pandemic, his wound care followup was less frequent than normal circumstances. At the initial follow-up visit in a few weeks later, the scalp mass was found to become a large protuberant lesion without any evidence of healing, which raised significant concern for a possible malignancy. Although computed tomography (CT) of the head was recommended for further evaluation, he did not follow up with his PCP for several months due to fear for contracting COVID-19.

He was eventually referred to the surgical oncology clinic until 5 months later from the initial presentation, at which point he was noted to have a large, ulcerated mass on the left scalp. The mass was not fixated to the scalp, and mobile on physical exam (Fig. 1). Biopsy of the lesion was obtained in the clinic under local anesthesia, which demonstrated malignant spindle cell neoplasm favoring leiomyosarcoma. A CT head demonstrated a mildly enhancing exophytic, left frontal scalp mass measuring $3.4 \times 3.3 \mathrm{~cm}$ with no evidence of invasion to the underlying bone (Fig. 2a, b). Staging CT scan of the chest, abdomen and pelvis revealed no obvious distant metastatic lesions.

\section{Treatment}

Wide local excision of the lesion was planned with possible myocutaneous rotation flap. He underwent a radical excision of left scalp lesion. The pericranium of the scalp was resected as a deep margin, and the $9 \times 7 \mathrm{~cm}$ scalp defect was reconstructed using a full-thickness skin graft harvested from the left supraclavicular area (Fig. 3a, b). The donor site was closed using an advancement flap measuring $15 \times 7 \mathrm{~cm}$.

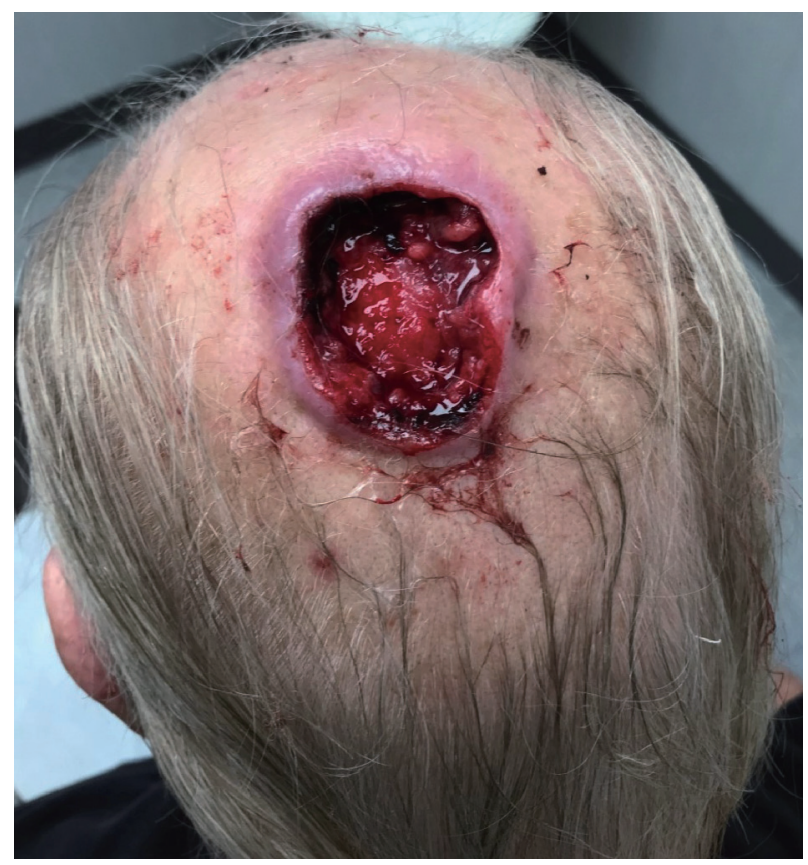

Figure 1. A representative image of the scalp mass of the presented patient. A large, ulcerated mass on the left scalp was noted, which was mobile and not fixated to the scalp.

home from the recovery. Final pathology demonstrated $5.4 \times$ $5.1 \times 2.2 \mathrm{~cm}$ grade 2 malignant spindle cells consistent with leiomyosarcoma (T3). A mitotic rate was 28/10 high-power field (HPF), and all margins were negative (Fig. 4a-c).

On his first postoperative visit, the patient was doing well with no surgical site occurrences. Subsequently, he is being considered for adjuvant radiation, given the tumor size, high mitotic rate, and grade 2 histology.

\section{Follow-up and outcomes}

\section{Discussion}

The patient tolerated the procedure well and was discharged

The exact pathophysiology of the development of leiomyo-
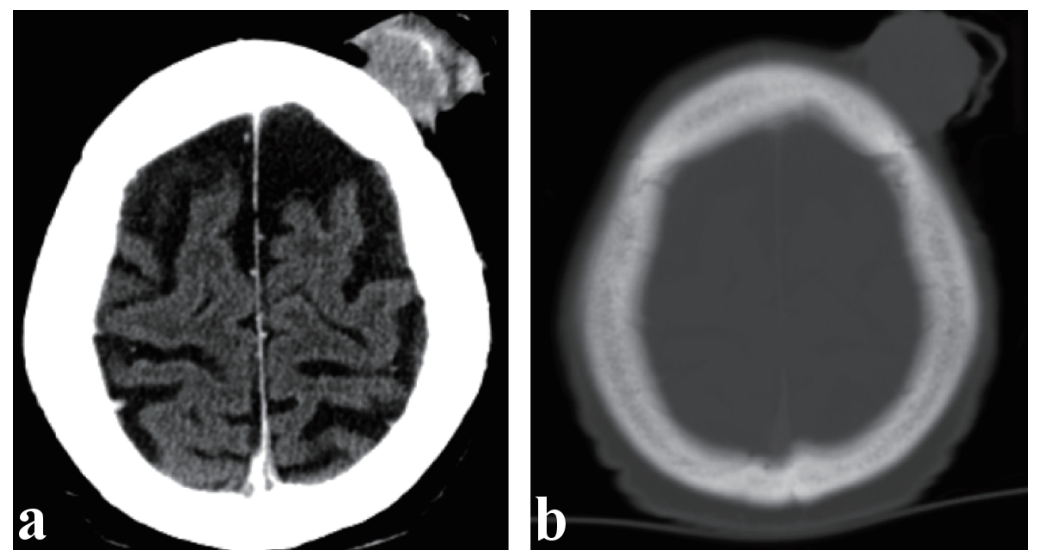

Figure 2. Representative computed tomography (CT) images of the presented patient. (a) Soft tissue window demonstrating a mildly enhancing exophytic left frontal scalp mass measuring $3.4 \times 3.3 \mathrm{~cm}$. (b) Bone window demonstrating no evidence of invasion to the underlying bone. 


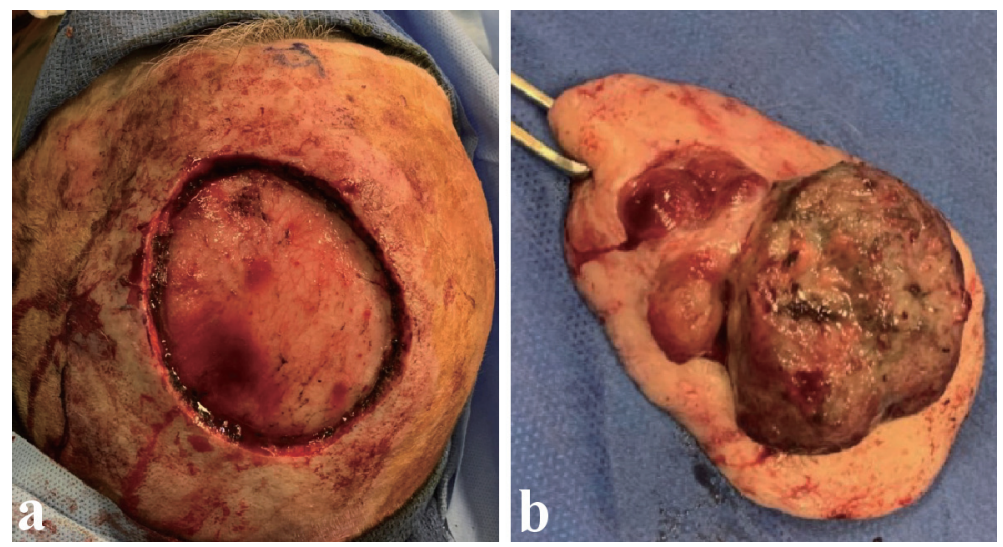

Figure 3. Representative images of the intraoperative findings of the presented patient. (a) Wide local excision of the scalp lesion was performed and the pericranium of the scalp was resected as a deep margin. (b) The gross finding of the specimen, which measured $5.4 \times 5.1 \times 2.2 \mathrm{~cm}$.

sarcomas remains unclear; however, it has been suggested that leiomyosarcomas are derived from either the arrector pili, cutaneously, or the smooth muscle wall of blood vessels, subcutaneously [5]. Some studies have found that cutaneous leiomyosarcomas tend to occur in patients with a history of radiation or trauma at the site; this trauma can include a tick bite, contusion, or burn [6]. Another common finding among patients with cutaneous leiomyosarcomas is that these sarcomas usually occur in hair bearing areas, like the scalp [7]. Although the cause of most sarcomas remains unclear, the patient in the presented case suffered direct tissue injury to his scalp prior to presentation.

Several case reports have discussed a potential link between the development of sarcoma and trauma due to the development of direct tissue damage and inflammation to the site [8-10]. It is theorized that failure to exit the healing phase leads to chronic inflammation, where inflammatory mediators like cytokines and growth factors are overactive, leading to tumorigenesis [10]. A recent study with a mouse model supports the connection between a history of injury at the site prior to sarcoma development [11]. In this study, tissue injury led to changes in muscle progenitors, specifically the HGF/c-MET signaling pathway, promoting sarcoma formation [11]. The activation of the inflammatory pathway may help explain the development of leiomyosarcoma at the site of injury in our patient.
Furthermore, leiomyosarcomas tend to be hemorrhagic and locally aggressive, as seen in the presented patient [2]. Although metastatic potential is low, local progression of these lesions is rather rapid. A superficial scalp leiomyosarcoma reportedly increased by $3.8 \mathrm{~cm}$ in diameter within 2 months [2]. They also can progress locally involving the underlying bone, which is only seen in $10 \%$ of cases [6]. Prompt diagnosis and treatment is crucial to improve overall survival from this challenging disease.

However, there may be some changes in presentation due to late presentation of the sarcoma amidst the pandemic owing to patients not seeking care routine case/follow-up during this time, and prioritizing COVID-19 care and prevention, as well as healthcare clinics limiting appointment availability and frequency. This makes prompt diagnosis even more critical requiring high index of suspicion when patients are seen by physicians.

Sarcoma management for curative intention is comprised of wide resection of the tumor with negative margin, and chemotherapy or radiation therapy in adjuvant or neoadjuvant fashion [8-10]. Cure of leiomyosarcoma depends on early diagnosis and prompt management; if the sarcoma is diagnosed before locally advanced state or distant metastasis, complete tumor excision has potential to be curative. It is also proposed that adjuvant radiotherapy may reduce risk of local recurrence

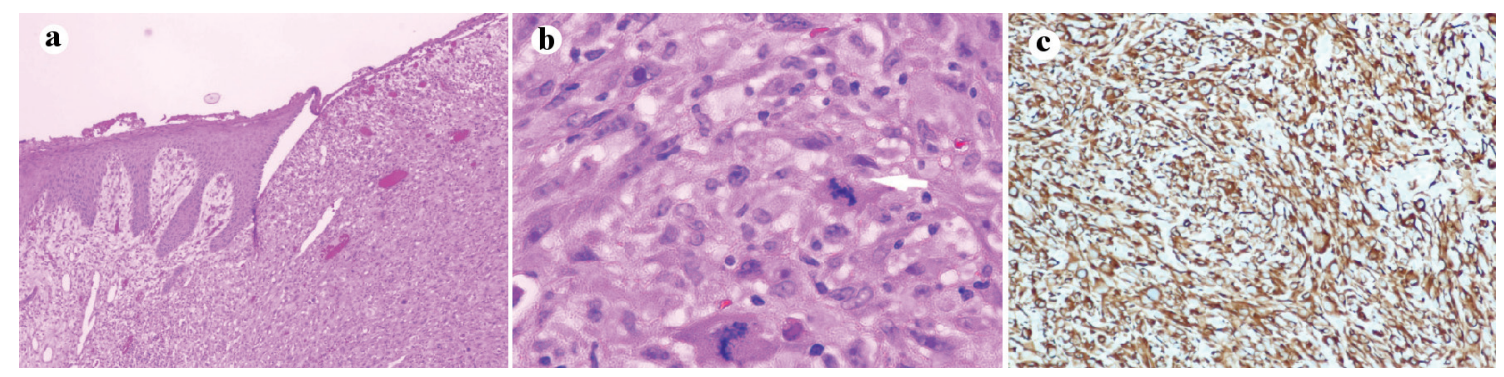

Figure 4. Pathology slides of the scalp leiomyosarcoma in the presented patient. (a) Low-power field ( $\times 10)$ : skin with ulceration and proliferation of neoplastic smooth muscles cells occupying the dermis. (b) High-power field $(\times 40)$ : neoplastic smooth muscle cells showing cytologic atypia, frequent mitosis and single cell apoptosis. (c) Medium-power field $(\times 20)$ with immunostain for desmin: the tumor is diffusely positive with desmin. 
in surgically treated head and neck leiomyosarcoma, even in the absence of positive margins [9]. Preoperative radiation has potential benefits for patients with larger tumors or locally advanced sarcomas [12], especially when the close margin resection is anticipated. Furthermore, it is hypothesized that tumor shrinkage may facilitate surgery and decrease the risk of intraoperative seeding of tumor cells [13]. Had our patient sought care earlier and if he had been able to have proper appointments with regular follow-up, the diagnosis may have been obtained much sooner. This could have led to prompt diagnosis and treatment, which, in his case, could have resulted in a less extensive resection.

Due to COVID-19, the healthcare system had to be modified to accommodate increasing volume of patients with $\mathrm{COV}$ ID-19 infection. Early in the pandemic, to lessen the load on hospitals, the Centers for Medicare and Medicaid Services suggest limiting all "non-essential" surgeries and procedures. An elective surgery acuity scale has been issued by the American College of Surgeons to help distinguish which patients should proceed to surgery based on urgency and patient health as precautions for patient safety from COVID-19 infection [14]. Specifically, for cancer care, the two fundamental issues at hand include physical outpatient visit will leave the patients vulnerable to possible exposure and cancer treatment may predispose them to more harmful effects of COVID-19. Prompt cancer diagnosis has been affected because of limited primary care visits as well as cancer screening procedures due to patient reluctance towards routine office visits and less capacity for non-COVID care [4]. This was seen in the presented patient who delayed his follow-up appointments for his scalp lesion, while he presented to his PCP for a potential COVID19-related symptoms of chest pain.

Patient reluctance towards routine office visits is particularly notable in the geriatric population. It took the presented patient 42 days to follow up with his PCP after his lesion was concerned for malignancy. These patients have greater risks and fear of COVID-19 exposure, leaving them more vulnerable to exacerbations of existing conditions, such as locally advanced cancer, as was the case for this patient leading to a more extensive resection. There is clear evidence supporting the claim that an increase in age is associated with higher rates of comorbidities with COVID-19, leading to this fear [15]. As a result of such panic, these patients are reluctant to schedule an appointment which is essential in diagnosing a treatable cancer. Additionally, patients are more likely to disregard mild symptoms during COVID-19 to avoid disease contraction. According to a meta-analysis studying the collateral effect of COVID-19 at a healthcare center in Italy, only 16 patients were diagnosed with a solid tumor during their 8-week lockdown. Compared to the number of diagnoses made in previous years during the same period, only $45.7 \%$ of the expected cases were seen [16]. The prediction is that the number of patients with solid tumors has remained the same; however, the number of patients visiting their physicians to document and diagnose the illness has dropped due to COVID-19 precautions.

COVID-19 constraints tend to defer face-to-face visits with physicians, due to inadequate physician protective equipment, lockdown, and risk of spread. As a result, virtual encounters are proving to be crucial in overcoming these limita- tions $[12,17]$. With telemedicine, potential disease exposure is minimized for both the patient and the healthcare provider, allowing patients to receive timely care. Virtual encounters help provide safe and convenient medical care by helping triage patients and providing continuity of care at a distance [18]. According to a study, the use of telemedicine services increased by at least $86 \%$ in the USA, excluding one hospital that saw an increase in consultations ranging from 2- 24\% [19]. However, some issues faced by this tool include lack of experience, funds, and infrastructure [20]. To strengthen our healthcare system, it is crucial that we improve telemedicine to be able to better manage future healthcare crises.

Both cancer and COVID-19 present great risk to patients; it is important to weigh the risks and benefits associated with cancer care during the pandemic. Although cancer treatment can leave patients more susceptible to COVID-19, delayed diagnosis and treatment will leave them immensely vulnerable to a potential cancer that may have been treated appropriately. Delaying in cancer diagnosis can lead to what may have been an early-stage disease presenting in an advanced stage with a grimmer prognosis, especially if the patient will only present once symptoms reach such a point that their concern overcomes their fear of the pandemic. What may have been a relatively straightforward lesion to treat may require more extensive and morbid treatments and care.

\section{Learning points}

In conclusion, we recently experienced a significantly delayed presentation of a scalp leiomyosarcoma requiring extensive excision and flap reconstruction due to the patient fear for COVID-19 pandemic. Both cancer and COVID-19 present great risk to patients; it is important to weigh the risks and benefits associated with cancer care during the pandemic.

\section{Acknowledgments}

We acknowledge great support from Dr. Kay and Dr. Arif, in the Department of Pathology at Mount Sinai South Nassau for providing pathological slides.

\section{Financial Disclosure}

Authors have no financial disclosure or funding related to the current study.

\section{Conflict of Interests}

Authors have no conflict of interest.

\section{Informed Consent}

The informed consent was obtained from the patient. 


\section{Authors Contributions}

Study concept: HH, AE and ES. Writing (draft): HH, AE and HT. Writing (review and editing): KT, HT and ES. Supervision: KT, RD, HT and ES.

\section{Data Availability}

The authors declare that data supporting the findings of this study are available within the article.

\section{References}

1. Pellitteri PK, Ferlito A, Bradley PJ, Shaha AR, Rinaldo A. Management of sarcomas of the head and neck in adults. Oral Oncol. 2003;39(1):2-12.

2. Cheung ST. Superficial leiomyosarcoma on the scalp - A rare cutaneous soft tissue tumor. J Am Acad Dermatol. 2007;56(2):AB56.

3. Hanna TP, Evans GA, Booth CM. Cancer, COVID-19 and the precautionary principle: prioritizing treatment during a global pandemic. Nat Rev Clin Oncol. 2020;17(5):268270.

4. Helsper CW, Campbell C, Emery J, Neal RD, Li L, Rubin $\mathrm{G}$, van Weert $\mathrm{H}$, et al. Cancer has not gone away: A primary care perspective to support a balanced approach for timely cancer diagnosis during COVID-19. Eur J Cancer Care (Engl). 2020;29(5):e13290.

5. Skoulakis C, Chimona TS, Tsirevelou P, Papadakis CE. Subcutaneus leiomyosarcoma of the neck: a case report. Cases J. 2010;3:52.

6. Pop M, Botar Jid C, Hotoleanu C, Vasilescu D, Sfrangeu S. Superficial leiomyosarcoma of the scalp: a case report. Med Ultrason. 2011;13(3):237-240.

7. Monsef ARM F, Farshchian M, Eghbalian F. Leiomyosarcoma of Scalp. Iran Red Crescent Med J. 2004:98-98.

8. Coley WB, Higinbotham NL. Injury as a causative factor in the development of malignant tumors. Ann Surg. 1933;98(6):991-1012.

9. Dijkstra MD, Balm AJ, Gregor RT, Hilgers FJ, Loftus BM. Soft tissue sarcomas of the head and neck associated with surgical trauma. J Laryngol Otol. 1995;109(2):126129.

10. Bar Y, Merimsky O. Soft-tissue sarcoma following traumatic injury: case report and review of the literature. Front Oncol. 2017;7:134.

11. Van Mater D, Ano L, Blum JM, Webster MT, Huang W, Williams N, Ma Y, et al. Acute tissue injury activates satellite cells and promotes sarcoma formation via the HGF/ c-MET signaling pathway. Cancer Res. 2015;75(3):605614.

12. Cheng EY, Dusenbery KE, Winters MR, Thompson RC. Soft tissue sarcomas: preoperative versus postoperative radiotherapy. J Surg Oncol. 1996;61(2):90-99.

13. Suit HD, Mankin HJ, Wood WC, Gebhardt MC, Harmon DC, Rosenberg A, Tepper JE, et al. Treatment of the patient with stage M0 soft tissue sarcoma. J Clin Oncol. 1988;6(5):854-862.

14. Yuen E, Fote G, Horwich P, Nguyen SA, Patel R, Davies J, Houlton J, et al. Head and neck cancer care in the COVID-19 pandemic: A brief update. Oral Oncol. 2020;105:104738.

15. Wang L, He W, Yu X, Hu D, Bao M, Liu H, Zhou J, et al. Coronavirus disease 2019 in elderly patients: Characteristics and prognostic factors based on 4-week follow-up. J Infect. 2020;80(6):639-645.

16. Chiaravalli S, Ferrari A, Sironi G, Gattuso G, Bergamaschi L, Puma N, Schiavello E, et al. A collateral effect of the COVID-19 pandemic: Delayed diagnosis in pediatric solid tumors. Pediatr Blood Cancer. 2020;67(10):e28640.

17. Chou E, Hsieh YL, Wolfshohl J, Green F, Bhakta T. Onsite telemedicine strategy for coronavirus (COVID-19) screening to limit exposure in ED. Emerg Med J. 2020;37(6):335-337.

18. Hollander JE, Carr BG. Virtually perfect? Telemedicine for Covid-19. N Engl J Med. 2020;382(18):1679-1681.

19. J SC, Alice K, M HA, Aaditya B, Jeff B, A CC. Systemwide implementation of telehealth to support military Veterans and their families in response to COVID-19: A paradigm shift. Journal of Military, Veteran and Family Health. 2020;6(S2):50-57.

20. Bokolo AJ. Exploring the adoption of telemedicine and virtual software for care of outpatients during and after COVID-19 pandemic. Ir J Med Sci. 2021;190(1):1-10. 